\title{
Aprender haciendo en lnvestigación como estrategia de aprendizaje.
}

\author{
María Cristina Gamboa Mora ${ }^{1}$ \\ Yenny García Sandoval ${ }^{2}$
}

\begin{abstract}
Resumen
Investigación realizada en el desarrollo de dos cursos académicos con la participación de 128 estudiantes. El objetivo fue analizar la metodología aprender haciendo, como alternativa de aprendizaje activo, que permitió sistematizar y realimentar el proceso en las aulas del campus virtual de las especializaciones de la Escuela Ciencias de la Educación de la Universidad Nacional Abierta y a Distancia, (UNAD), durante el primer período académico de 2011. Las categorías de análisis y su articulación con las líneas de investigación, se seleccionaron por método inductivo. Como resultado de este proceso investigativo, se organizaron sistemáticamente 43 rutas de investigación y 55 proyectos, a través de la validación y construcción colectiva que confirman la eficiencia de la metodología planteada y el auge de la investigación que adelantan los maestros sobre su práctica pedagógica. La categoría en la cual se inscriben el mayor número de propuestas de investigación corresponde a la implementación de estrategias didácticas con herramientas Web 2.0 y Web 3.0, con el fin de optimizar las practicas educativas e incorporar las Tecnologías de la Información y la Comunicación, TICs, en las aulas.
\end{abstract}

Palabras clave: investigación, estrategia de aprendizaje, autoaprendizaje, trabajo en equipo, aprendizaje social, método de enseñanza.

\footnotetext{
1. Licenciada en Química y Biología de la Universidad de la Salle, Especialista en Análisis de datos de la Universidad de la Salle y Magíster en docencia de la Química de la universidad Pedagógica Nacional. Estudiante de Doctorado en Innovación e Investigación en didáctica. Universidad Nacional de Educación a Distancia (UNED). Auditora Interna GP1000:MECI, certificada por ICONTEC. maria.gamboa@unad.edu.co 


\title{
Learning by doing in research as a learning strategy
}

\begin{abstract}
This was a research conducted during the development of two academic terms with the participation of 128 students. Its aim was to analyze the methodology of learning by doing, as an alternative active learning, as well as to systematize and to feedback this process at the National Open and Distance Education Institute - UNAD - , during first academic period 2011. The categories of analysis and their articulation with research lines were selected by an inductive method. As a result of this research process, 43 routes and 55 research projects were systematically organized, through validation and collective construction confirming the efficiency of the proposed methodology and the increasing importance of research by teachers on their own teaching practice. Thus, the category in which the largest number of research proposals are inscribed is the implementation of teaching strategies with Web 2.0 and Web 3.0, intended to optimize educational practices and to incorporate information and communication technologies, TIC's in classrooms.
\end{abstract}

Key words: research, learning strategy, self instruction, team work, social learning, teaching method.

\section{Introducción}

La educación superior en Colombia debe responder a un sistema globalizado, en el cual la tecnología brinda diversas herramientas que se transforman asertivamente en estrategias pedagógicas con base en el conocimiento disciplinar, pedagógico y didáctico.

Las perspectivas pedagógicas y didácticas, es decir, el conjunto de saberes que enmarcan a la educación como fenómeno social y humano, evolucionan y los medios y las mediaciones que facilitan el proceso de enseñanza-aprendizaje avanzan a la par con las Tecnologías de la Información y la Comunicación, TIC. Lo anterior permite afirmar, que en la modalidad de educación a distancia 
y la metodología virtual se imponen los propósitos de globalización porque promueven a gran velocidad el trabajo colaborativo y cooperativo.

Es así como la creación de redes con base en las TIC, constituyen una de las herramientas de cooperación que le permitirá a la Universidad crecer a través de la reflexión, la innovación y el intercambio mutuo, convirtiéndose en una institución exigente, flexible que responde a las necesidades de la población, enriquecida por la discusión académica, descentralizada e interconectada lo que favorece la creación de una cultura más diversa, responsable con la humanidad, por lo anterior, construida en la transparencia porque tiene como base el debate y la validación de las comunidades académicas a nivel global.

La administración del diseño curricular supone para los docentes el reto de llevar al aula (en este caso virtual), los contenidos y las competencias que definen su materia de enseñanza y transformarlos mediante los medios y recursos didácticos, a través de estrategias metodológicas, en aprendizajes significativos para los estudiantes que se forman bajo su dirección (Castillo y Cabrerizo, 2006).

Además del aspecto curricular, es necesario reconocer que "históricamente el centro de la educación a distancia lo ocupa el estudiante, sujeto activo y responsable, capaz de encargarse autónomamente de su propio aprendizaje, empleando para ello diversas estrategias desarrolladas con el apoyo de diferentes mediaciones y medios pedagógicos”, (Herrera Sánchez, 2005).

Ahora bien, uno de los aspectos en discusión permanente en la educación, es lo referente a los trabajos de grado, que en el ámbito colombiano suponen el acercamiento de los estudiantes a los procesos de investigación, pero su relevancia debería ser leída en un contexto amplio reafirmando su importancia (Torres Soler, 2005) afirmaba:

"Las capacidades investigativas de los estudiantes se fortalecen sólo a través de la investigación misma, del aprender-haciendo, en un ambiente de trabajo colectivo para la búsqueda de alternativas, donde prime la interdisciplinaridad, la colaboración y la armonía de trabajo en equipo, como la tolerancia y el respeto a la diferencia".

Teniendo en cuenta lo anterior, es relevante anotar que experiencias previas con respecto a los cursos seminario de investigación y trabajo de grado en las aulas virtuales, hicieron pertinente revisar su optimización a través del replanteamiento de su estructuración de manera tal que, los estudiantes de las especializaciones de la Escuela Ciencias de la Educación de la Universidad Nacional Abierta y a Distancia se aproximen a los procesos de investigación y puedan presentar sus proyectos como alternativas de grado. Se trata entonces, de aproximar a los estudiantes a una metodología pedagógica basada en la 
investigación-acción, en donde la acción crea las condiciones óptimas para el aprendizaje convirtiéndolo en un proceso gratificante y efectivo.

Los elementos estructurantes de la metodología aprender haciendo son: acción, método y teoría. Las autoras han definido dicha metodología para los espacios académicos virtuales de investigación, en correspondencia con los lineamientos del reglamento estudiantil de la Universidad Nacional Abierta y a Distancia, UNAD: "los elementos estructurantes de la propuesta educativa de la UNAD, son: el estudio independiente, el trabajo colaborativo y el acompañamiento tutorial" (Herrera Sánchez, 2005). En la figura 1 se refieren los elementos para la propuesta de aprender haciendo en investigación, articulando la teoría y la praxis:

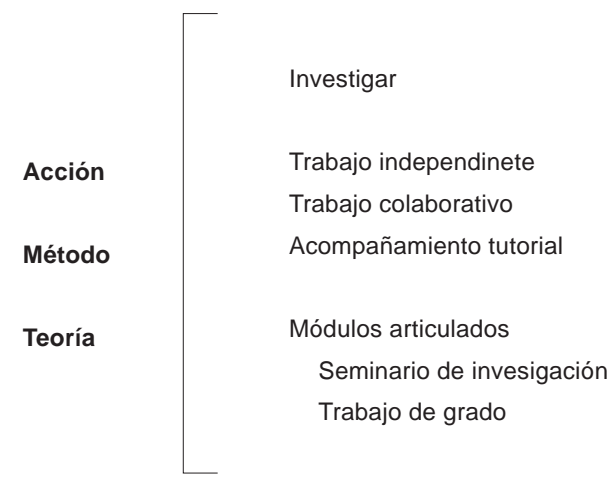

Figura 1. Elementos estructurantes de aprender haciendo.

Carballo (2005) se refiere a los elementos estructurantes como la fórmula mágica compuesta por tres elementos entrelazados y priorizados; se aprende interrelacionando la acción, la teoría y la experiencia. Lo aprendido se consolida, amplia y generaliza mediante la teoría la cual corresponde al saber acumulado que es poco eficiente sin la acción. En este sentido el aprendizaje activo es el proceso por medio del cual se aprende haciendo. Como Herrera Sánchez (2005) señalaba, es la experiencia educativa donde se centra la atención en la metodología de estudio y desde la cual, más que conocimiento, se generan habilidades, destrezas, actitudes y hábitos de pensamiento y de acción con el propósito de ampliar las fronteras del saber y saber hacer, para desarrollar la competencia fundamental del aprender haciendo.

En concordancia con lo anterior, el proceso de aprendizaje es viable por la activación de procesos cognitivos; dichos procesos se activan a través de las estrategias pedagógicas, que se seleccionan en el proceso de planeación pedagógica y didáctica, la cual parte de la reflexión sobre las concepciones y prácticas docentes, el diseño y la consecución de acciones y la sinergia del aula, todo lo anterior, con el fin de transformar las prácticas tradicionales de enseñanza-aprendizaje por prácticas de calidad para la educación colombiana, para lo cual: "se requiere establecer relaciones entre los 
procesos cognitivos, las estrategias pedagógicas y didácticas seleccionadas, las metas establecidas por competencias y la organización jerarquizada de dominios conceptuales", (Gamboa, Briceño y Sánchez, 2009).

Los procesos de investigación en UNAD y específicamente en la Escuela de Ciencias de la Educación, plantean la necesidad de articular a la investigación los procesos académicos y curriculares, en el marco de la investigación formativa, cuyas acciones están orientadas hacia la formación de los tutores en metodología y epistemología, que permitan la configuración de los cursos y módulos hacia la búsqueda del desarrollo del espíritu investigativo en los estudiantes, promoviendo la generación de iniciativas de investigación y de semilleros en los cuales se fomente el trabajo en redes intra e institucionales. Retomado lo expuesto en una investigación previa: "las actividades de investigación en el aula, favorecen la motivación en los estudiantes, son un estímulo en su proceso de formación y además favorecen la autoestima, la automotivación, la construcción colectiva del conocimiento y el trabajo en equipo",(Gamboa, et al., 2009).

Otro aspecto revisado, lo constituye el trabajo colaborativo en los foros de discusión; frente al mismo, Gunawardena, Lowe y Anderson (1997) propusieron el modelo para el análisis de la construcción social del conocimiento en tres fases: Comparación de la información, donde se comparten y argumentan puntos de vista sobre un tema, disonancia e inconsistencia, se identifican acuerdos y desacuerdos los cuales se aclaran a través de preguntas y respuestas, Negociación o co-construcción, se clarifican significados, se plantean acuerdos y se presenta una propuesta conjunta. Prueba de lo co-construido, se somete a discusión la nueva construcción se contradice a través de la argumentación y se replantea o no la propuesta conjunta. Finalmente, Acuerdos y aplicaciones, con base en la discusión argumentada, la reflexión en grupo y el consenso, se define la propuesta y se socializa el concepto elaborado.

\section{Metodología}

La presente investigación definió seis fases para analizar la metodología de aprender haciendo, en las cuales se realizan análisis de corte cualitativo y cuantitativo que permiten evaluar la efectividad del proceso académico.

\section{La población}

Ciento veintiocho estudiantes de especializaciones en educación de la Escuela Ciencias de la Educación, a nivel nacional, inscritos en los cursos académicos seminario de investigación y trabajo de grado en modalidad a distancia, metodología virtual, profesionales en diversas áreas del conocimiento. 


\section{Fases del diseño aprender haciendo}

En la figura 2, se describen las fases de la propuesta y sus elementos de categorización de acuerdo con las características de cada una, dentro del proceso académico.

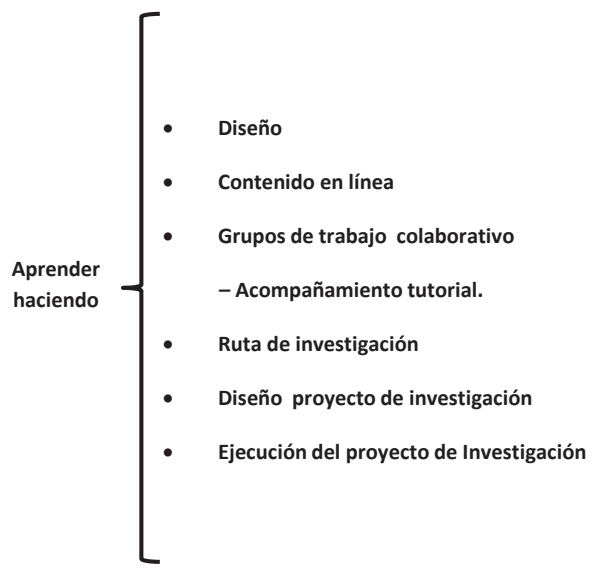

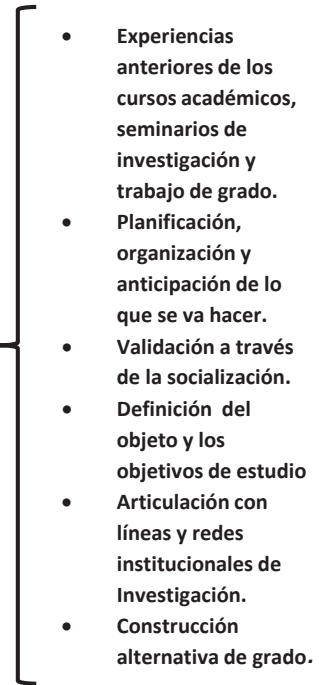

Figura 2. Fases de aprender haciendo para las aulas virtuales de la UNAD.

Fase uno, diseño a través de la administración del currículo: la evaluación continua del currículo conllevó a la modificación de la propuesta de los cursos seminario de investigación y trabajo de grado, con el propósito de hacer investigación a través de los cursos académicos. Con base en las experiencias previas, se articulan los contenidos de los cursos académicos, teniendo como presupuesto que la teoría, en la metodología aprender haciendo permite ampliar, generalizar y consolidar lo aprendido.

Fase dos, diseño contenidos en línea: la teoría se presenta a los estudiantes en un contenido en línea desarrollado bajo exelearning, el cual se desarrolla con base en el diseño de los cursos académicos, se implementa con medios y mediaciones que apoyan el proceso. Se distribuyen los contenidos en tres capítulos para el curso académico Seminario de Investigación y dos capítulos, para el curso académico trabajo de grado porque el curso es de dos créditos. Los contenidos se articulan de tal forma, que con el seminario de investigación pueda construirse un objeto de investigación contextualizado y pertinente junto con objetivos viables y oportunos para el tipo de trabajo seleccionado por el estudiante. En el seminario trabajo de grado se pretende trabajar con los insumos obtenidos en el seminario de investigación y ajustarlo en términos metodológicos, iniciando su implementación. 
Fase tres, grupos de trabajo colaborativo y acompañamiento tutorial: el trabajo colaborativo en general, pretende la construcción colectiva de conocimiento teniendo como base la discusión que se sustenta a través de la argumentación. $\mathrm{Al}$ interior de los grupos se argumentan, negocian, aprueban o se modifican las posturas de los participantes y en particular se busca constituirlo como el espacio de validación a través de la socialización, tal y como se hace en las comunidades académicas, cumpliendo la propuesta de aprender haciendo en investigación.

Todos los foros como apoyo a su desarrollo contienen una guía y una rúbrica de evaluación, con el propósito de acompañar a los estudiantes en su proceso para que conozcan desde el inicio no solo cómo se realizará la evaluación del proceso, sino también el horizonte de trabajo que se propone. La rúbrica le permite a los estudiantes conocer todos y cada uno de los parámetros que se tendrán en cuenta para su evaluación, dándole significado a la discusión para la construcción en colectivo.

Fase cuatro, rutas de investigación: los estudiantes a través del foro, validan su objeto y sus objetivos de investigación, finalmente, los estudiantes del curso seminario de investigación presentan la ruta para plantear el proyecto, el cual se articula a una línea de investigación en el curso trabajo de grado, articulándose la formación en investigación.

Para la validación de las rutas de investigación se encontraron como categorías emergentes las experiencias de aula, el diseño y la aplicación de herramientas Web 2.0 y 3.0, y proyectos con impacto social, político o cultural, seleccionadas a través del método inductivo que permiten llegar a conclusiones generales. El proceso se describe en la figura 3.

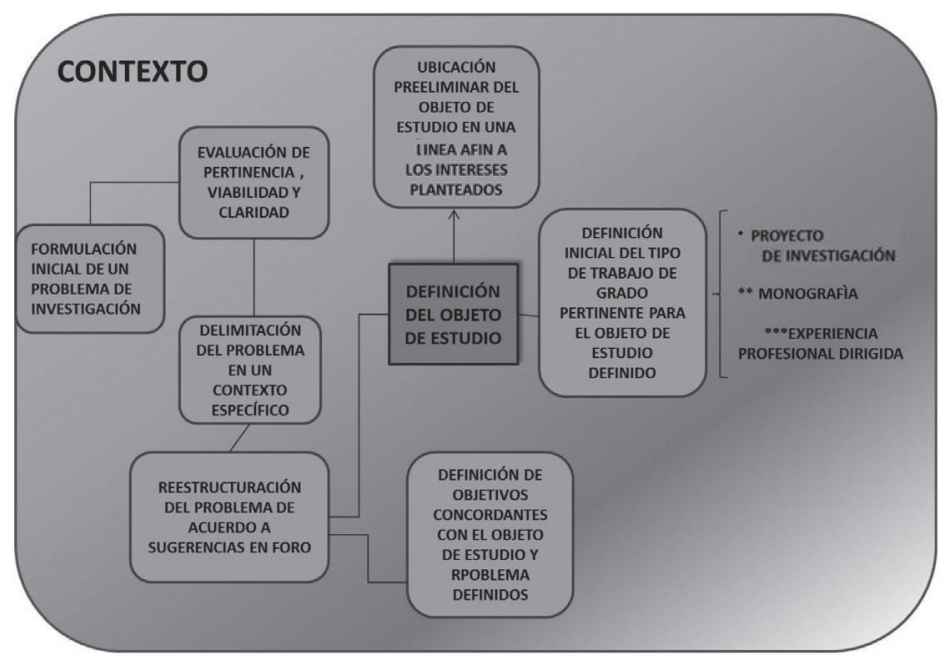

Figura 3. Ruta de investigación. 
Los estudiantes en el curso de trabajo de grado, en la fase inicial, complementan la ruta diseñada y validada en seminario de investigación, seleccionando una de las alternativas de grado descritas en la figura 4, que les permitirá acceder al título de especialista previa aprobación de los créditos correspondientes.

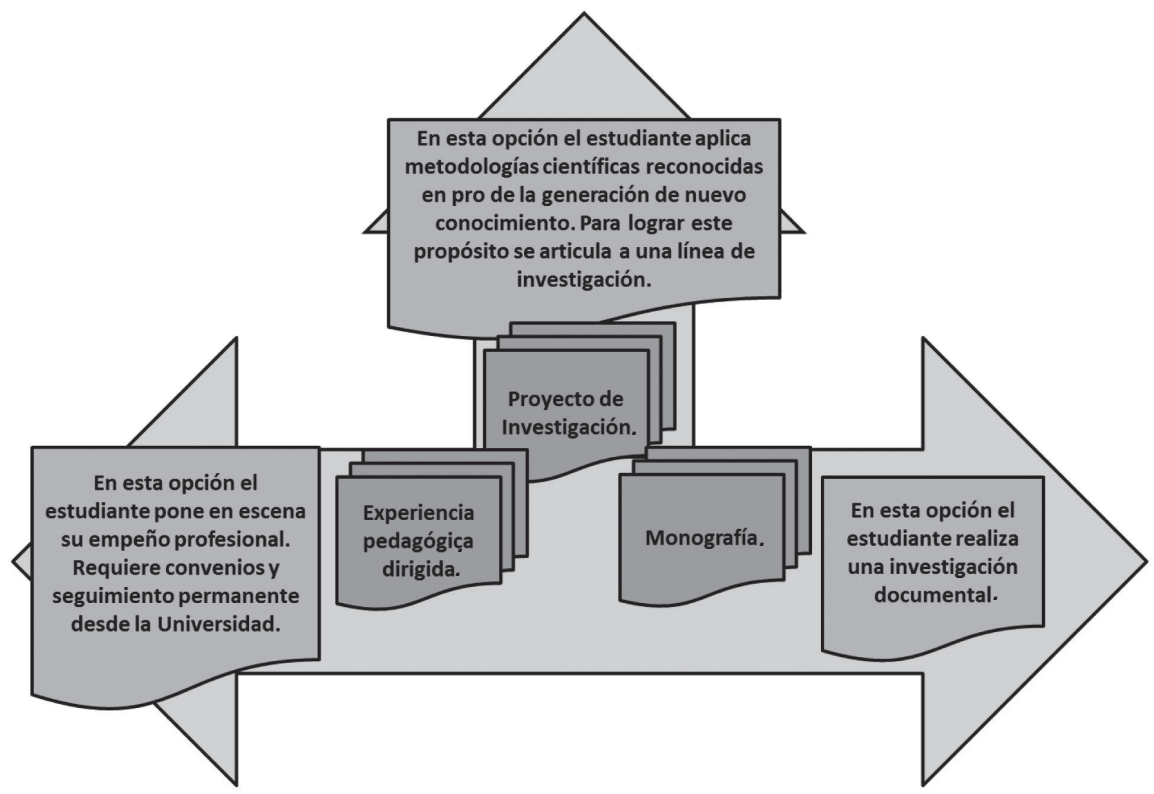

Figura 4. Alternativas de grado para las Especializaciones - UNAD.

Fase cinco, diseño proyectos de investigación: los estudiantes en el foro inicial del curso académico trabajo de grado, en compañía de su tutor validan la articulación de las propuestas de investigación con líneas de investigación. Las líneas de investigación propias de la Escuela Ciencias de la Educación, implican diversas temáticas relacionadas con este campo del saber como lo muestra la figura 5. La validación se lleva a cabo en los foros de trabajo colaborativo a través de la socialización y discusión entre pares.

Fase seis, ejecución del proyecto de investigación: los estudiantes al finalizar el curso académico trabajo de grado tienen un documento base con el cual el comité curricular de las especializaciones determina la asignación de un asesor de trabajo de grado para finalizar el proceso, el cual se describe en la figura 6. 


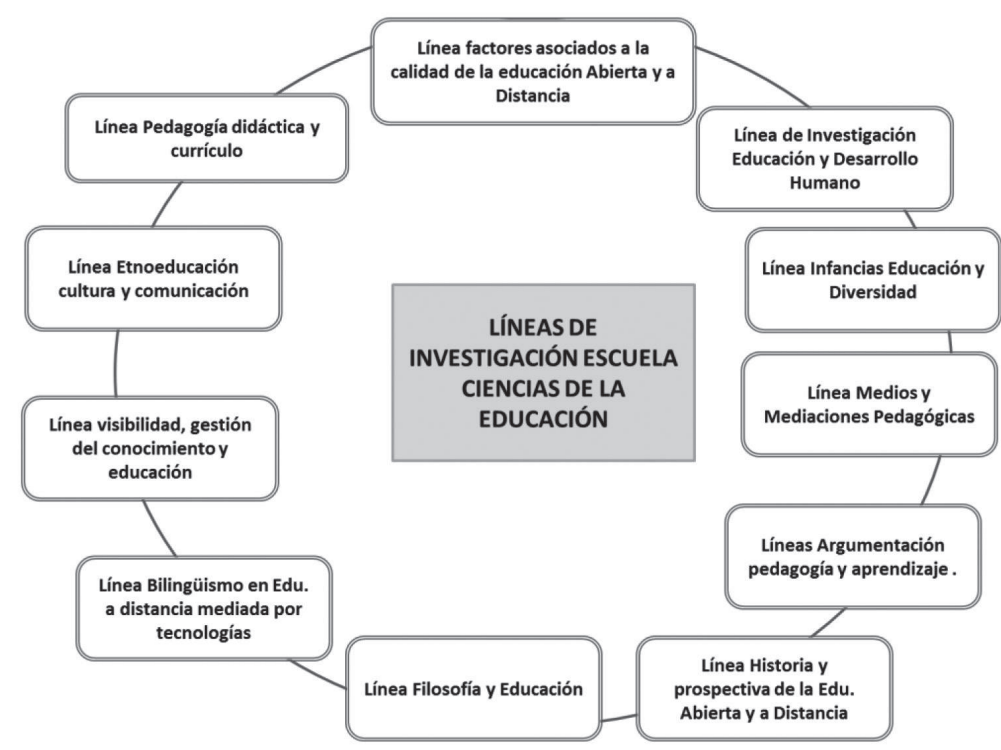

Figura 5. Líneas de Investigación Escuela Ciencias de la Educación - (ECEDU)

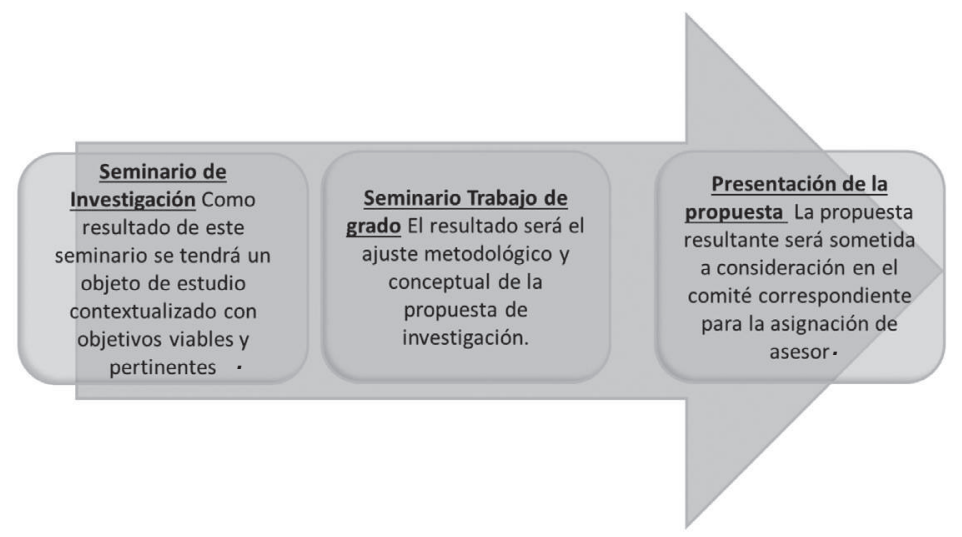

Figura 6. Esquema de aprobación de grado espcializaciones ECEDU - UNAD.

\section{Resultados}

\section{Participación cobertura y aprobación del proceso}

Participaron en el estudio 128 estudiantes en el proceso de articulación de los seminarios de investigación y de trabajo de grado, de los cuales, 6 desertaron, 19 
estudiantes no aprobaron, 103 aprobaron el proceso. Los resultados de los estudiantes con base en la participación se muestran en la figura 7.

\section{Participantes de la investigación}

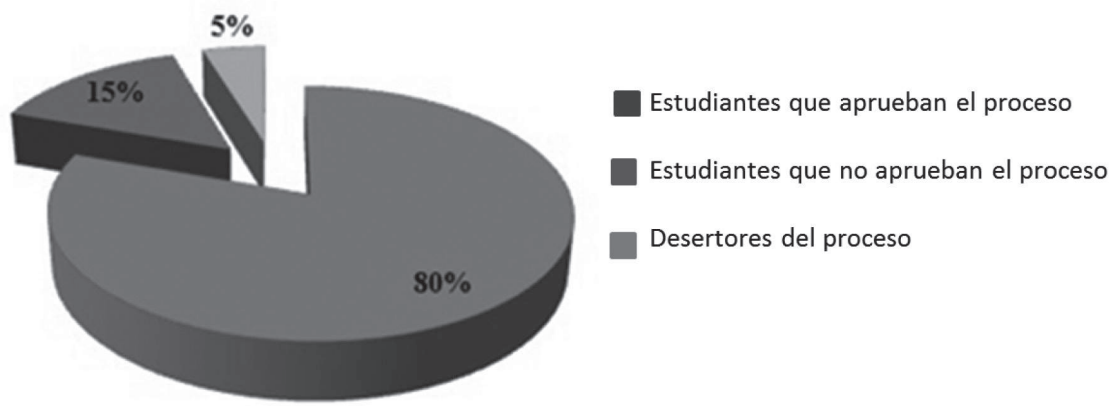

Figura 7. Participantes de la Investigación.

Se organizaron 43 rutas de investigación en seminario de investigación y 55 proyectos; de estos últimos, 40 proyectos correspondientes al $72.7 \%$, ejecutaron la propuesta una vez validados los instrumentos diseñados. Cabe aclarar que todo el proceso se realizó en el aula virtual.

\section{El diseño curricular y los contenidos en línea}

Las unidades didácticas para los dos cursos fueron articuladas de tal forma, que se pudiera dar continuidad al trabajo planteado inicialmente en el seminario de investigación como ruta de investigación (definición de objeto de estudio contextualizado y objetivos) y ajustado en los referentes metodológicos en el seminario trabajo de grado. La articulación permite al estudiante contar con las herramientas conceptuales para actuar en este proceso de investigar, a través del trabajo colaborativo, el trabajo independiente y el acompañamiento tutorial.

El archivo EXE que presenta la teoría de los cursos académicos, cumple con los elementos y taxonomía del protocolo académico definido por la Vicerrectoría de Medios y Mediaciones Pedagógicas de la UNAD, Salazar (2009) que contiene ficha técnica, introducción, justificación, intencionalidades formativas, unidades didácticas, contexto teórico, metodología, sistema de evaluación y fuentes documentales. El archivo vincula al estudiante con objetos virtuales de aprendizaje, páginas especializadas y fuentes bibliográficas diversas; el desarrollo de la propuesta permitió evidenciar las ventajas de la integración de los dos cursos sin disminución alguna de los lineamientos exigidos por la universidad optimizando la efectividad de los mismos, al tiempo que disminuye el marasmo de los estudiantes al fomentar el desarrollo de propuestas grupales. 


\section{Grupos de trabajo colaborativo y acompañamiento tutorial}

El trabajo colaborativo el trabajo independiente y el acompañamiento tutorial, son decisivos en la acción para el proceso de investigación. Este es el método por excelencia que se implementa para investigar a través de las aulas virtuales. La socialización y validación, son las estrategias para la construcción y coconstrucción social del conocimiento. Los foros, permiten trascender la comunicación unidireccional y en este caso posesiona al estudiante, como par evaluador de los trabajos puestos a consideración por los estudiantes del grupo asignado, de tal forma que se promueve el desarrollo del pensamiento crítico, autonomía y respeto.

Todos los foros de trabajo colaborativo en las aulas virtuales tienen guía de actividades y rúbricas de evaluación.

\section{Ruta de Investigación}

Los participantes del estudio validan 43 rutas de investigación y al finalizar la articulación de los cursos como estrategia de aprendizaje, se obtienen los proyectos consolidados se distribuyen por alternativas de grado.

\section{Distribución alternativas de grado de los estudiantes participantes en el proyecto}

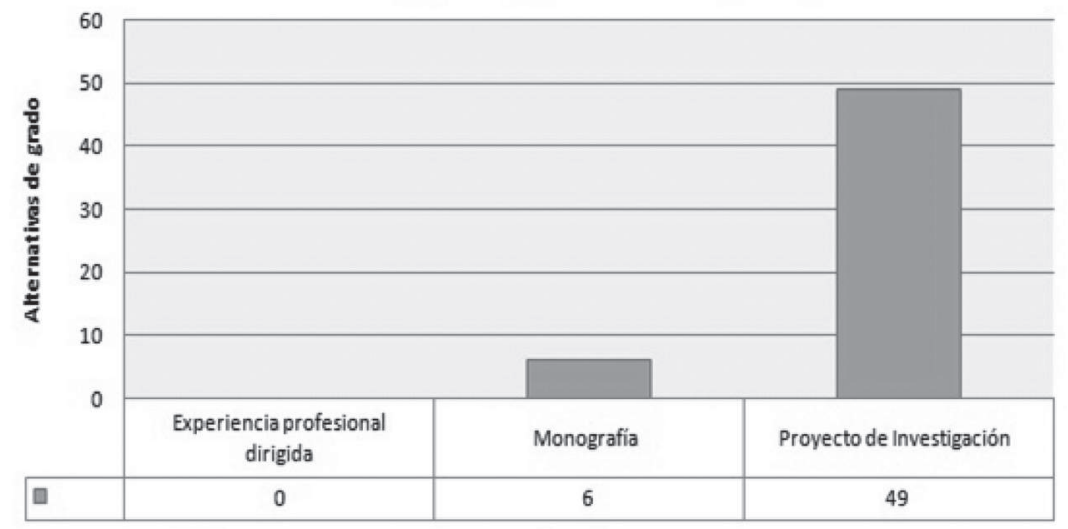

Figura 8. Distribución de proyectos por categorías emergentes.

Es interesante evidenciar cómo no se presentaron propuestas para experiencias dirigidas; sin embargo, en los proyectos de investigación, la propia experiencia de aula de los estudiantes cuando trabajan como docentes, se constituye en elemento de investigación, lo cual implica indagar a futuro, la dimensión y las ideas alternativas que los estudiantes dan a cada una de las alternativas de grado. 


\section{María Cristina Gamboa y Yenny García Sandoval}

Aprender haciendo en Investigación como estrategia de aprendizaje. Artículo producto de la investigación.

\section{Diseño y ejecución de los proyectos de Investigación}

Con base en la socialización y validación al interior de los foros de trabajo colaborativo, se construyen proyectos de investigación que permiten plantear tres categorías emergentes, resultado de los aspectos temáticos seleccionados para la definición del objeto de estudio, como se muestra en la tabla 1.

Tabla 1. Categorías emergentes

\begin{tabular}{|c|c|}
\hline Categorías & Definición \\
\hline Experiencias de aula & $\begin{array}{l}\text { Esta categoría corresponde a los proyectos plan- } \\
\text { teados que tienen como objeto de estudio la prác- } \\
\text { tica docente que realiza el estudiante, aquí, el } \\
\text { contexto propio de aula donde él se desenvuelve, } \\
\text { se constituye en materia prima para trazar proce- } \\
\text { sos que tienden a plantear el diagnóstico o carac- } \\
\text { terización de diversas situaciones particulares, } \\
\text { a diseñar estrategias para resolver problemas, } \\
\text { mejorar procesos de argumentación o plantear } \\
\text { procesos didácticos que promueven el desarrollo } \\
\text { autónomo en el aula. }\end{array}$ \\
\hline Experiencias de aula & $\begin{array}{l}\text { Este tipo de experiencias son en sí, una réplica } \\
\text { del aprender haciendo, al posesionar al estudian- } \\
\text { te como investigador de la realidad del aula, in- } \\
\text { vestigando hechos educativos que beneficiarán } \\
\text { no solo su práctica sino que, le brindan herramien- } \\
\text { tas para dimensionar el alcance y complejidad de } \\
\text { un proceso de investigación, esto es congruente } \\
\text { con lo argumentado a continuación: "la teoría, el } \\
\text { saber ya acumulado, es poco eficiente y es pre- } \\
\text { ciso utilizarlo como un punto de contrastación, de } \\
\text { comprobación, de aseguramiento. Se aprende } \\
\text { interrelacionando acción y teoría, experiencia y } \\
\text { saberes acumulados, pero para aprender la prio- } \\
\text { ridad está en la acción, en la experiencia" (Carba- } \\
\text { llo, 2006, 2). }\end{array}$ \\
\hline Diseño y aplicación de herramientas Web 2.0 y 3.0 & $\begin{array}{l}\text { En esta categoría se ubican las situaciones plan- } \\
\text { teadas en torno al diseño y uso de herramientas } \\
\text { web } 2.0 \text { y } 3.0 \text { en entornos de educación formal, no } \\
\text { formal e informal. En este caso se incluyen entre } \\
\text { otros, el uso de redes sociales, en donde se bus- } \\
\text { ca no solo reconocer las diversas formas como } \\
\text { dichas redes funcionan e inciden en las dinámicas } \\
\text { de comunicación actual, sino que también se pro- } \\
\text { pende por plantear propuestas para optimizar su } \\
\text { uso en ámbitos educativos, otro aspecto aborda- } \\
\text { do es el mejoramiento de procesos de comunica- } \\
\text { ción con la utilización de este tipo de herramientas } \\
\text { (blogs, wikispace, Second Life, Open sim, entre } \\
\text { otros) y el diseño de material web para ser aplica- } \\
\text { do en el abordaje de diversas temáticas (diseño e } \\
\text { implementación de web sites). }\end{array}$ \\
\hline
\end{tabular}




\begin{tabular}{|l|l|}
\hline Categorías & Definición \\
\hline Proyectos con impacto social, político o cultural & $\begin{array}{l}\text { Esta categoría incluye todos los proyectos ten- } \\
\text { dientes a diagnosticar las causas, efectos o las } \\
\text { alternativas de intermediación en situaciones } \\
\text { problema que se refieren a asuntos de carácter } \\
\text { social, como el consumo de drogas, el manejo de } \\
\text { procesos de impacto ambiental, otros de carácter } \\
\text { político como violencia y desplazamiento, políti- } \\
\text { cas educativas, políticas de contratación, dere- } \\
\text { chos humanos, entre otros. Además, se incluyen } \\
\text { proyectos de carácter cultural como procesos de } \\
\text { identidad cultural, liderazgo, por mencionar solo } \\
\text { algunos. Cabe anotarse que estos proyectos bus- } \\
\text { can de una u otra forma incidir sobre la realidad } \\
\text { y escenarios que vivencian los estudiantes, para } \\
\text { entenderlos, transformarlos, dinamizarlo por ru- } \\
\text { tas alternativas que impliquen reconocer lo que } \\
\text { ofrece el contexto. }\end{array}$ \\
\\
\end{tabular}

La distribución de los proyectos en las categorías emergentes puede observarse en la figura 9.

\section{Distribución de proyectos de investigación por categorías emergentes}

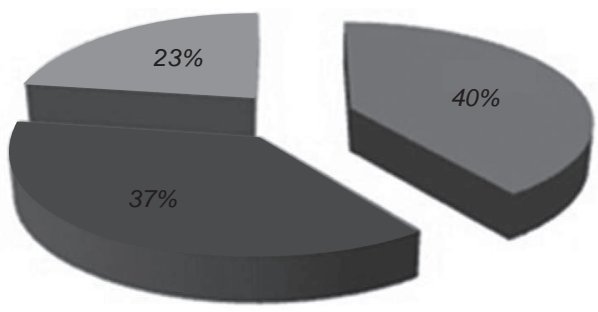

Experiencias de aula.

Estrategias Web 2.0 o Web 3.0

Impacto social, político y cultural.

Figura 9. Distribución de proyectos por categorías emergentes.

\section{Articulación de los proyectos con las líneas de investigación}

Los proyectos deben tener una clara articulación con las líneas de investigación de la Escuela Ciencias de la Educación, lo cual puede llegar a redundar (en el desarrollo de los proyectos) en una optimización de los mismos, dado que estos pueden, de diversas maneras, contribuir en el enriquecimiento conceptual de dichas líneas, enrutando de mejor forma las temáticas que en su interior se plantean. La distribución de los proyectos en las líneas puede verse en la figura 10.

Se plantea entonces la necesidad de reconocer la importancia de una evaluación constante de los procesos educativos desarrollados al interior de un programa 
de formación, tendientes no solo a revisar de forma introspectiva su desarrollo, sino a plantear alternativas de optimización, que integren diversos elementos como las líneas de investigación, la articulación entre espacios académicos ofrecidos y la integración de estudio independiente, el trabajo colaborativo y el acompañamiento tutorial en un equilibrio tal, que dinamice y flexibilice el rol de docente y estudiante.

\section{Distribución de proyectos de investigación por líneas de la escuela ECEDU}

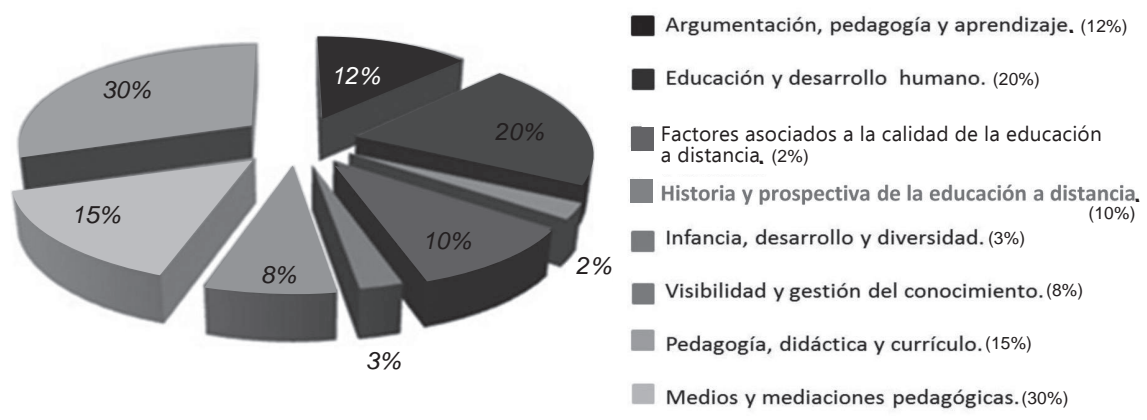

Figura 10. Distribución de proyectos por línea de investigación.

\section{Conclusiones}

Se considera que la metodología de aprender haciendo es efectiva en este caso, si los estudiantes logran definir la ruta de investigación en seminario de investigación, validan la propuesta en los foros colaborativos del curso seminario trabajo de grado, y si se privilegia la alternativa proyecto de investigación dentro de las alternativas de grado, como evidentemente sucedió al presentarse 49 proyectos de investigación por parte de los estudiantes de las especializaciones de la Escuela Ciencias de la Educación

Las categorías emergentes mostraron una clara tendencia de los estudiantes a plantear proyectos de investigación en lo referente a experiencias de aula y el diseño e implementación de estrategias web 2.0 y 3.0, (77\%), lo cual puede deberse en el primer caso al reconocimiento de la práctica docente como objeto de investigación, evidenciando que la cercanía con el entorno brinda elementos para plantear problemas contextualizados, en el segundo caso, el mismo trabajo realizado en los seminarios en el aula virtual le permitió a los estudiantes optimizar sus prácticas, incorporando el uso de los ciberlugares, contextos interactivos y el uso de herramientas web. 
La metodología de aprender haciendo hace aportes a la formación de los maestros como investigadores de su práctica pedagógica, facilita la implementación de TIC en el aula y brinda herramientas para asumir el papel de sujeto activo en los procesos de investigación.

La administración del diseño curricular supone la evaluación continua por parte de estudiantes y tutores, convirtiéndose en la herramienta que permite modificar las estrategias metodológicas y los contenidos para generar aprendizajes significativos.

Implementar la metodología de aprender haciendo en investigación es una alternativa eficiente ya que los estudiantes en promedio para el año 2010 tardaban un año en el proceso de asesoría de trabajo de grado, dado que en el curso académico trabajo de grado se definía la ruta de investigación. Con los resultados de este proyecto de investigación se evidencia que la metodología de aprender optimiza el proceso de aprendizaje y pueda disminuirse en seis meses el proceso de asesoría para los proyectos pendientes de aplicar instrumentos y en algunos casos reportar resultados $(27.3 \%)$.

La interacción de calidad referida a una permanente tutoría y participación, en los foros de trabajo colaborativo permite que el método, la teoría y la acción arrojen resultados positivos en el proceso.

Hay una contribución por parte de los proyectos a las líneas de investigación de la Escuela, dado que ofrecen referentes respecto a la dirección que pueden tomar las temáticas de investigación referidas por dichas líneas, además los proyectos aportaron al $72.7 \%$ de las líneas (8 líneas de 11 existentes), mostrando la pertinencia de la metodología.

Los resultados obtenidos evidencian la importancia de trabajar la investigación articulando diversos actores en el proceso, esto implica que a futuro además de involucrar a las líneas de investigación, se impliquen y nutran las redes con los resultados de los proyectos de los estudiantes, consolidando los semilleros que al interior de dichas redes entretejen el tejido que soporta un sistema de investigación.

\section{Para la reflexión y la discusión}

La continuidad de los procesos de investigación dentro del aula requiere de voluntad política para que los actores involucrados dediquen tiempo a este importante proceso; la dedicación por parte de los maestros, demanda tiempos de gestión importantes para que los resultados relevantes de su práctica pedagógica se puedan divulgar y validar ante la comunidad científica, y es allí donde se deben aunar esfuerzos para que la administración del sistema educativo y sus actores inviertan lo necesario para alcanzar estándares de calidad en los diferentes niveles educativos y en las diversas modalidades de enseñanza. 
Los docentes como investigadores en el aula crean constantemente propuestas que pueden hacer eficiente el proceso de enseñanza-aprendizaje en los diferentes niveles del sistema, para este caso en particular, en el nivel superior, las políticas educativas deben trazarse no solo pensando en la cobertura de la educación que si bien es cierto, es una política de inclusión necesaria para la época, lo es quizás en mayor grado, una educación con calidad. Para avanzar en la calidad de la educación es importante conseguir que los estudiantes reciban un verdadero acompañamiento de sus docentes y tutores, para que se enriquezca el proceso en todos los aspectos y eso requiere de los recursos administrativos que les apoya.

La sistematización de las experiencias docentes también logra importantes aportes para la construcción colectiva de estrategias didácticas pertinentes, métodos y metodologías que fortalezcan los procesos educativos. Un número adecuado de estudiantes garantiza el acompañamiento, el seguimiento y la construcción organizada de conocimiento, la educación a distancia virtual favorece no solo la construcción de conocimientos sino la apropiación de las Tecnología de la Información y la Comunicación, TIC, y la construcción de comunidades de aprendizaje por eso cada día cobra mayor importancia esta metodología y la disponibilidad de recursos para garantizar la calidad de la educación.

Es aquí dónde, a través de la siguiente pregunta planteamos el punto para la reflexión:

¿Estamos dedicando el tiempo suficiente que se requiere para implementar sistemas educativos de calidad y los sistemas garantizan los recursos para ello?

\section{Referencias bibliográficas}

Carballo, R. (2006). Aprender haciendo. Guía para profesores. Aproximación a los espacios de aprendizaje basados en la acción, la experiencia y el grupo de trabajo y aplicaciones prácticas. Segundo encuentro sobre experiencias grupales innovadoras en la docencia universitaria. Disponible en: http://www.ucm.es/info/tropico/EGlo2/ PONENCIAS/carballo.pdf [Consulta 2012, 15 de junio]

Castillo, S y Cabrerizo, J. (2006). Formación del profesorado en Educación superior. Volumen II. España: Mc.Graw Hill/Interamericana de España, S.A.U.

Gamboa, M. Briceño, J y Sánchez, D. (2009). La planeación pedagógica como estrategia de investigación para fortalecer la formación cientifica de los estudiantes en las áreas de ciencias básicas de la Universidad Manuela Beltrán. Revista Tecné, episteme y didaxis. TEA. 826-832. 
Gamboa, M. (2011). Curso Académico Trabajo de grado. 207018. Bogotá: ECEDU-UNAD.

Gamboa, M. (2011). Curso Académico. Seminario de Investigación. 202030. Bogotá: ECEDU-UNAD

Gunawardena, Ch., Lowe, C. y Anderson, T. (1997). Analysis of a global online debate and the development of an interaction analysis model for examing social construction of knowledge in computer conferencing. Journal of Educational Computing Research, $17(4), 395-429$

Herrera, G. (2005). Curso: Trabajo Académico a Distancia. Bogotá: Universidad Nacional Abierta y a Distancia, UNAD.

Salazar, R. (2009). Referentes para el desarrollo de contenidos didácticos. Vicerrectoria de Medios y Mediaciones Pedagógicas. Bogotá: Universidad Nacional Abierta y a Distancia, UNAD.

Torres, C. (2005). Para qué los semilleros de investigación. Disponible en: http:// www.revistamemorias.com/edicionesAnteriores/8/semilleros.pdf [Consulta 2012, 15 de junio] 